\title{
Migración y desarrollo económico en México: un análisis factorial de correspondencias
}

\author{
JOSÉ CÉSAR LENIN NAVARRO CHÁVEZ* \\ FRANCISCO VENEGAS-MARTÍNEZ** \\ AMÉRICA IVONNE ZAMORA TORRES***
}

RESUMEN: Este trabajo analiza el fenómeno migratorio en México por entidad federativa y su interrelación con los niveles de desarrollo económico durante 2009. Para ello se utiliza el Análisis Factorial de Correspondencias (AFC) a fin de examinar la correlación de los estados con la migración, el desarrollo económico y el Programa $3 \times 1$. La evidencia empírica indica que los mayores niveles de desarrollo se encuentran en el Distrito Federal, Nuevo León, Querétaro y Jalisco; mientras que con el menor nivel de desarrollo están Chiapas, Guerrero y Oaxaca. Asimismo, Jalisco, el Estado de México, Michoacán, Guanajuato y Distrito Federal sobresalen con la más alta migración; en tanto que Campeche, Tlaxcala, Baja California Sur, Yucatán y Tabasco presentan la más baja influencia migratoria.

PALABRAS CLAVE: análisis factorial de correspondencias, migración, desarro1lo, programa $3 \times 1$, México.

* Profesor-investigador del Instituto de Investigaciones Económicas y Empresariales de la Universidad Michoacana de San Nicolás de Hidalgo, México.

**Profesor-investigador de la Escuela Superior de Economía del Instituto Politécnico Nacional, México.

*** Profesora-investigadora del Instituto de Investigaciones Económicas y Empresariales de la Universidad Michoacana de San Nicolás de Hidalgo, México. 
ABSTRACT: This paper analyzes the phenomenon of migration in Mexico by state and its interrelation with the levels of economic development in 2009. To do this, the Correspondence Factor Analysis (CFA) is used for examining the correlation between states, migration, economic development, and $3 \times 1$ programs. Empirical evidence shows that the states with higher levels of development are Distrito Federal, Nuevo León, Querétaro, and Jalisco, while the lowest development is found in Chiapas, Guerrero and Oaxaca. Also Jalisco, Estado de Mexico, Michoacán, Guanajuato, and Distrito Federal stand out with the highest migration, while Campeche, Tlaxcala, Baja California Sur, Yucatán, and Tabasco display the lowest migratory influence.

KEY WORDS: factorial correspondence analysis, migration, development, $3 \times 1$ program, Mexico. 


\section{INTRODUCCIÓN}

71 fenómeno de la migración ha sido estudiado desde muchas perspectivas. Por ejemplo, la interrelación entre migración y remesas ha sido examinada en Guatemala y Pakistán por Adams (1991, 1998 y 2005), en China por Rozelle et al. (1999) y en México, en el caso de Procampo, por Sadoulet et al. (2001). Por su parte, Lucas (1987) estudia la relación entre migración y desarrollo en África. Asimismo, el tema de migración y desigualdad en México ha sido tratado por McKenzie y Rapoport (2005). Por último, la interrelación entre el mercado laboral y la migración es estudiada, por ejemplo, en los trabajos de Munshi (2003) y Mora y Taylor (2005), entre otros.

En México, los crecientes flujos migratorios y la importancia que las remesas han adquirido como fuente de divisas del país hacen necesario estudiar el vínculo que se establece entre la migración y el crecimiento del producto interno bruto (PIB), así como entre la migración y el bienestar social. La necesidad de contar con una metodología que permitiera asociar estas variables, así como otras relacionadas con el fenómeno migratorio, y que además permitiera identificar aquellas entidades federativas que guardan ciertos niveles de homogeneidad, condujo a trabajar con el Análisis Factorial de Correspondencias (AFC). El AFC consta de cuatro fases principales: el cálculo de una matriz que exprese la dependencia entre las variables, la extracción del número óptimo de factores, la rotación de soluciones para facilitar su interpretación y la estimación de las puntuaciones gráficamente donde en cada etapa será necesario tomar decisiones para la ejecución adecuada del análisis factorial.

El presente trabajo utiliza el AFC destacando sus especificidades metodológicas y contrastándolo con la evidencia empírica sobre la migración en México. En términos metodológicos se encuentran los siguientes elementos: las comunalidades y el gráfico de sedimentación de las variables, la matriz de componentes y componentes rotados, el gráfico de componentes en espacios rotados en tres dimensiones y el gráfico de puntuaciones por estados y variables. 
El objetivo de la investigación es identificar los principales indicadores que dan cuenta de la relación entre migración y desarrollo para México durante 2009, a partir de la instrumentación metodológica del Análisis Factorial de Correspondencias.

En el estudio se consideraron 21 indicadores: población (habitantes), migración internacional (miles de pesos), migración internacional como porcentaje, remesas (miles de pesos), remesas como porcentaje, Índice de Desarrollo Humano (IDH), índice de salud, índice de educación, índice de ingreso, posición del PIB, PIB per cápita, PIB, tasa de desempleo, población económicamente activa (PEA), inflación, tasa de analfabetismo, $3 \times 1$ federal, $3 \times 1$ estatal, $3 \times 1$ municipal, $3 \times 1$ migrantes y $3 \times 1$ total.

La información obtenida fue para las 32 entidades federativas tomando como año de estudio el 2009. Las fuentes de la información fueron el Instituto Nacional de Estadística, Geografía e Informática (INEGI) a través del sistema de cuentas nacionales y la encuesta nacional de ocupación y empleo, el Informe sobre Desarrollo Humano, Secretaría de Desarrollo Social (Sedesol), la Secretaría de Educacuión Pública (SEP) y el Banco de México (Banxico).

\section{Análisis Factorial de Correspondencias}

Al realizar un estudio sobre cualquier temática en el área de las ciencias sociales, el investigador generalmente busca una combinación de variables cuantitativas y cualitativas, cosa que puede hacer el estudio más complejo y más rico, lo cual permite que los resultados e interpretaciones sean más realistas, debido a esto se han desarrollado técnicas de análisis multivariado, como los métodos factoriales y de clasificación. Véase, por ejemplo, Pérez (2006). ${ }^{1}$

El Análisis Factorial es una técnica estadística multivariada cuyo objetivo es la definición de una estructura subyacente en una matriz de datos. El análisis factorial permite estudiar la estructura de las correlaciones existen-

${ }^{1}$ El Análisis Factorial de Correspondencias, ideado por el estadístico francés Benzecri, en 1973, fusiona al Análisis de Proximidades con el Análisis de Componentes Principales logrando así un análisis de similaridad (Callealta, 2005).

$126 \frac{\text { PRIMER SEMESTRE } 2014}{\text { MIGRACIÓN Y DESARROLLO NÚM. } 22}$ 
tes en un gran número de variables y casos (estados de la República), definiendo un número de dimensiones comunes subyacentes, denominadas componentes. Por medio de esta metodología se estudia la dependencia entre las variables, la asociación entre atributos permitiendo una revisión de la intensidad de las atracciones y repulsiones entre las modalidades que pueden presentar las características cualitativas a partir del estudio de las frecuencias conjuntas observadas (véase Miquel et al., 1997).

Entre sus ventajas el análisis factorial, a diferencia de los análisis de regresión, no impone restricciones a priori sobre las formas funcionales, ni clasifica de antemano a las variables, sino que por el contrario el propio análisis va descartando aquellas variables que no son representativas y va agrupando a las variables en factores (ICTEM, 2011).

Adicionalmente, del análisis de atracción-repulsión entre modalidades de atributos (variables) diferentes, la técnica del AFC también permite realizar estudios de proximidad (similaridad/disimilaridad) entre las modalidades de una misma variable, es decir, permite evaluar la homogeneidad o sustituibilidad de las mismas. Para esto, se realiza la proyección de las modalidades sobre un espacio métrico en el que se aplica el Análisis de Componentes Principales para facilitar la interpretación causal simple de los comportamientos de similitud-atracción (Kim y Mueller, 1978). Los objetivos de la técnica, de acuerdo con Callealta (2005), son 1) descubrir las relaciones de atracción-repulsión entre las variables; 2) identificar las relaciones de proximidad existentes entre las distintas modalidades de una misma variable cualitativa; y 3) visualizar y caracterizar de forma simple las relaciones anteriores en un espacio con dimensión lo más reducida posible.

El análisis de la similaridad (proximidad) existente entre las modalidades de un atributo (variable), representadas por las respectivas distribuciones de frecuencias, condicionadas, a su vez, por la distribución de modalidades de otro atributo, nos permite analizar la homogeneidad de éstos en dos espacios de dimensiones diferentes entre los que se emplea la distancia de Benzecri, ${ }^{2}$ la cual es una ponderación entre los puntos inversamente propor-

${ }_{2}^{2}$ Distancia que recibe el nombre de CHI-CUADRADO, debido a que su expresión coincide con la prueba del mismo nombre que tradicionalmente se ha utilizado para comprobar la depen- 
cional a sus frecuencias (Rodríguez et al. 2003). El análisis de la asociación entre modalidades de los dos atributos diferentes será el resultado de conectar estos dos espacios y, en consecuencia, proyectarlos en un espacio común tridimensional donde la proximidad será interpretada como atracción y el alejamiento como repulsión, aplicando para esto el Análisis de Componentes Principales (Kruskal y Wish, 1981). Este es uno de los métodos más empleados dentro del análisis factorial, el cual permite formar un índice compuesto que sea capaz de representar un grupo de variables (Kennedy, 1985).

Comunalidades y Gráfico de Sedimentación de las Variables

Al realizar el AFC se deben seguir pasos que demuestren la confiabilidad de los resultados obtenidos, para lo cual la tabla de comunalidades es una herramienta útil, ya que permite identificar que parte de la varianza o dispersión de la variable se está logrando reproducir, mostrando la representatividad de las variables. Si el nivel de extracción que muestra la tabla de comunalidades es menor a 0.5 , significa que la variable a estudiar no se está explicando bien dentro del modelo, debido a que no tiene un nivel importante de representación, entre más cercana a uno esté la variable, mejor representada estará, por lo que el modelo mismo hace la selección de variables justo en esta primer etapa del análisis. En nuestro caso se observa que la columna de extracción muestra un grado de representación significativo para todas las variables, siendo el menor de 0.716 correspondiente a la variable $3 \times 1$ municipios; mientras que el valor más elevado, 0.991, corresponde a la variable Índice de Desarrollo Humano (IDH). Todas las variables se encuentran bien representadas dentro del modelo, pero es de mencionarse que en la parte alta se ubican MIGRACIÓN, REMESAS, I-INGRESO (índice de ingreso) y el PIB PER CÁPITA. En tanto que, en la parte baja se localizan la T-DESEMPLEO, $3 \times 1 \mathrm{EDO}, 3 \times 1 \mathrm{CTOTAL}$, PIB, I-SALUd (índice de salud) y POBLACión (véase cuadro 1).

dencia estocástica entre variables (Batista y Joan, 1997).

$128 \frac{\text { PRIMER SEMESTRE } 2014}{\text { MIGRACIÓN Y DESARROLLO NÚM. } 22}$ 
CUADRO 1

Tabla de Comunalidades

\begin{tabular}{lcc}
\hline & INICIAL & EXTRACCIÓN \\
\hline Población & 1.000 & 0.882 \\
Migración & 1.000 & 0.979 \\
Migración\% & 1.000 & 0.979 \\
Remesas & 1.000 & 0.978 \\
Remesas \% & 1.000 & 0.977 \\
IDH & 1.000 & 0.991 \\
I-Salud & 1.000 & 0.881 \\
I-Educación & 1.000 & 0.922 \\
I-Ingreso & 1.000 & 0.971 \\
Posición PIB & 1.000 & 0.881 \\
PIB per cápita & 1.000 & 0.960 \\
PIB & 1.000 & 0.875 \\
T-Desempleo & 1.000 & 0.756 \\
PEA & 1.000 & 0.948 \\
Inflación & 1.000 & 0.894 \\
T-Analfabetismo & 1.000 & 0.883 \\
$3 \times 1$ Federal & 1.000 & 0.913 \\
$3 \times 1$ Edo & 1.000 & 0.815 \\
$3 \times 1$ Municipio & 1.000 & 0.716 \\
$3 \times 1$ Migrantes & 1.000 & 0.832 \\
$3 \times 1$ Ctotal & 1.000 & 0.821 \\
\hline
\end{tabular}

Método de extracción: Análisis de componentes principales.

Fuente: Elaboración propia con base en los resultados obtenidos del AFC.

Otra medida importante de confiabilidad es el número de componentes o dimensiones que se van a considerar para representar las variables sujetas a análisis. Cada dimensión va a representar a las variables en cierta proporción, siendo las primeras dimensiones las que van a tener un mayor grado de representación, esto se expresa en la Gráfica de Sedimentación como una mayor distancia en el eje vertical a cero. El Gráfico de Sedimentación originalmente propuesto por Cattell (1966) es una representación gráfica del tamaño de los autovalores, permite ver si se está dejando fuera algún componente importante (Cox y Cox, 1994).

La dimensión número uno en nuestro estudio va a representar el $36.119 \%$ de la dispersión de las variables, la segunda dimensión el 20.498\%, la tercera 
el $8.409 \%$ y así sucesivamente (ver gráfica 1). Con base en estos resultados se consideran ocho componentes, ya que de esta manera se alcanza una representación de $89.772 \%$ de las variables, permitiendo que el estudio sea confiable.

\section{GRÁFICA 1}

Gráfico de Sedimentación

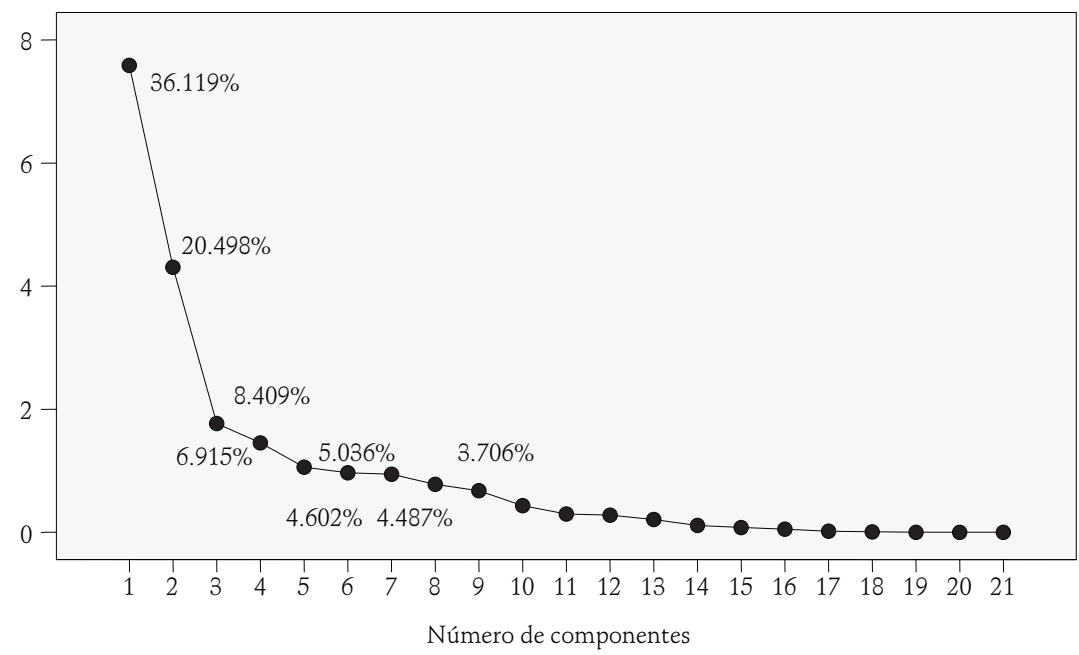

Fuente: Elaboración propia con base en los resultados obtenidos del Análisis Factorial de Correspondencias.

\section{Matriz de Componentes y Componentes Rotados}

Los primeros resultados del AFC se encuentran en la Matriz de Componentes y la Matriz de Componentes Rotados, que indican la dirección en el espacio donde se ubican las variables. Los signos positivo y negativo muestran el espacio en la dimensión donde se posicionan las variables, así como también sus niveles de correlación.

La Matriz de Componentes nos permite identificar en qué dimensión se encuentran mejor explicadas las variables. En nuestro estudio tenemos que en

$130 \frac{\text { PRIMER SEMESTRE } 2014}{\text { MIGRACIÓN Y DESARROLLO NÚM. } 22}$ 
el primer componente (dimensión) las variables mejor explicadas son: el IDH, I-INGRESO, I-EDUCACIÓN, POSICIÓN PIB y el PIB PER CÁPITA. En el segundo componente están la POBLACIÓN, el PIB y la MIGRACIÓN. En el tercero solamente aparece $3 \times 1$ ESTADOS. En el cuarto componente se localizan la INFLACIÓN y el $3 \times 1$ MUNICIPIOS. En el quinto se encuentra la PEA. En el séptimo se tiene a la TASA DE ANALFABETISMo y el $3 \times 1$ federal. En el sexto y octavo componentes no existen variables representativas (véase cuadro 2).

CUADRO 2

Matriz de Componentes

\begin{tabular}{|c|c|c|c|c|c|c|c|c|}
\hline & \multicolumn{8}{|c|}{ COMPONENTE } \\
\hline & 1 & 2 & 3 & 4 & 5 & 6 & 7 & 8 \\
\hline Poblacion & -0.223 & 0.799 & -0.383 & 0.061 & 0.165 & 0.074 & -0.050 & 0.094 \\
\hline Migracion & -0.586 & 0.748 & 0.221 & -0.040 & -0.035 & 0.001 & 0.105 & -0.114 \\
\hline Migracion\% & -0.586 & 0.748 & 0.221 & -0.040 & -0.035 & 0.001 & 0.105 & -0.114 \\
\hline Remesas & -0.692 & 0.695 & 0.007 & -0.054 & 0.045 & 0.077 & 0.064 & 0.015 \\
\hline Remesas \% & -0.694 & 0.694 & 0.004 & -0.054 & 0.043 & 0.075 & 0.062 & 0.012 \\
\hline Idh & 0.918 & 0.300 & 0.208 & 0.072 & -0.034 & 0.086 & -0.004 & 0.027 \\
\hline I-Salud & 0.742 & 0.259 & 0.450 & -0.144 & -0.051 & -0.168 & -0.090 & -0.025 \\
\hline I-Educacion & 0.860 & 0.276 & 0.182 & -0.117 & 0.013 & -0.217 & -0.112 & -0.021 \\
\hline I-Ingreso & 0.870 & 0.285 & 0.163 & 0.183 & -0.049 & 0.252 & 0.059 & 0.054 \\
\hline Posición Pib & -0.857 & -0.143 & -0.090 & -0.210 & 0.117 & -0.151 & -0.078 & 0.173 \\
\hline Pib Per Cápita & 0.830 & 0.359 & 0.117 & 0.212 & 0.005 & 0.249 & -0.008 & 0.144 \\
\hline Pib & 0.280 & 0.780 & -0.189 & 0.168 & 0.159 & 0.216 & -0.144 & 0.178 \\
\hline T-Desempleo & 0.656 & 0.370 & 0.121 & -0.117 & -0.022 & -0.370 & 0.004 & 0.152 \\
\hline Pea & 0.126 & -0.201 & 0.294 & -0.313 & 0.726 & 0.295 & -0.098 & -0.292 \\
\hline Inflación & 0.259 & -0.079 & -0.167 & 0.639 & 0.226 & -0.077 & 0.356 & -0.448 \\
\hline T-Analfabetismo & 0.340 & 0.155 & -0.081 & -0.129 & 0.340 & -0.404 & 0.628 & 0.214 \\
\hline $3 \times 1$ federal & 0.015 & -0.278 & 0.210 & -0.411 & -0.263 & 0.475 & 0.562 & 0.105 \\
\hline $3 \times 1$ edo & -0.367 & -0.256 & 0.641 & 0.011 & 0.355 & -0.027 & -0.091 & 0.261 \\
\hline $3 \times 1$ municipal & -0.417 & -0.169 & 0.460 & 0.515 & -0.110 & 0.079 & 0.053 & 0.126 \\
\hline $3 \times 1$ migrantes & -0.578 & -0.247 & 0.211 & 0.527 & 0.124 & -0.032 & 0.038 & 0.311 \\
\hline $3 \times 1$ ctotal & -0.470 & 0.269 & 0.558 & 0.010 & -0.250 & -0.211 & -0.002 & -0.331 \\
\hline
\end{tabular}

Método de extracción: Análisis de componentes principales. 8 componentes extraídos.

Fuente: Elaboración propia con base en los resultados obtenidos del AFC.

Si bien la Matriz de Componentes en un primer momento permite identificar las variables mejor explicadas, presenta algunos problemas de defini- 
ción de estas variables, que se resuelven en un segundo momento al trabajar con la Matriz de Componentes Rotados. Tal es el caso de la REMESAS, donde no se establece con claridad si su mejor representación se localiza en el componente uno o dos, pero que a través de esta última Matriz se sitúa a las REMESAS en el componente dos con un valor de 0.943 (véase cuadro 3).

\section{CUADRO 3}

Matriz de Componentes Rotados

\begin{tabular}{|c|c|c|c|c|c|c|c|c|}
\hline & \multicolumn{8}{|c|}{ COMPONENTE } \\
\hline & 1 & 2 & 3 & 4 & 5 & 6 & 7 & 8 \\
\hline Poblacion & 0.018 & 0.763 & -0.210 & -0.412 & 0.031 & -0.116 & 0.263 & 0.050 \\
\hline Migracion & -0.093 & 0.955 & 0.089 & 0.217 & -0.044 & -0.027 & -0.002 & -0.012 \\
\hline Migracion\% & -0.093 & 0.955 & 0.089 & 0.217 & -0.044 & -0.027 & -0.002 & -0.012 \\
\hline Remesas & -0.271 & 0.943 & 0.064 & -0.017 & -0.092 & -0.037 & 0.023 & -0.018 \\
\hline Remesas \% & -0.274 & 0.942 & 0.061 & -0.016 & -0.091 & -0.040 & 0.026 & -0.020 \\
\hline Idh & 0.963 & -0.155 & -0.152 & -0.079 & 0.071 & 0.022 & -0.014 & 0.072 \\
\hline I-Salud & 0.846 & -0.119 & -0.116 & 0.274 & -0.159 & 0.111 & 0.061 & 0.146 \\
\hline I-Educacion & 0.841 & -0.188 & -0.288 & 0.100 & -0.079 & 0.055 & 0.186 & 0.209 \\
\hline I-Ingreso & 0.930 & -0.128 & -0.078 & -0.204 & 0.166 & -0.008 & -0.115 & -0.010 \\
\hline Posición Pib & -0.817 & 0.249 & 0.184 & 0.045 & -0.323 & 0.041 & 0.080 & 0.051 \\
\hline Pib Per Cápita & 0.921 & -0.060 & -0.048 & -0.303 & 0.115 & -0.018 & -0.029 & -0.004 \\
\hline Pib & 0.526 & 0.522 & -0.142 & -0.496 & 0.029 & -0.049 & 0.234 & -0.025 \\
\hline T-Desempleo & 0.686 & -0.034 & -0.212 & 0.081 & -0.157 & -0.116 & 0.205 & 0.390 \\
\hline Pea & 0.040 & -0.117 & -0.037 & -0.013 & 0.022 & 0.963 & -0.055 & -0.004 \\
\hline Inflación & 0.114 & -0.149 & 0.058 & -0.020 & 0.906 & 0.005 & 0.133 & 0.122 \\
\hline T-Analfabetismo & 0.199 & -0.004 & -0.106 & -0.085 & 0.136 & 0.015 & -0.074 & 0.895 \\
\hline $3 \times 1$ federal & -0.032 & -0.118 & -0.011 & 0.038 & -0.121 & 0.051 & -0.936 & 0.053 \\
\hline $3 \times 1$ edo & -0.167 & -0.004 & 0.687 & 0.186 & -0.299 & 0.429 & 0.002 & 0.077 \\
\hline $3 \times 1$ municipal & -0.143 & 0.078 & 0.766 & 0.175 & 0.122 & -0.109 & -0.077 & -0.198 \\
\hline $3 \times 1$ migrantes & -0.415 & 0.052 & 0.793 & -0.064 & 0.082 & -0.088 & 0.093 & -0.018 \\
\hline $3 \times 1$ ctotal & -0.076 & 0.482 & 0.236 & 0.711 & -0.027 & -0.023 & 0.004 & -0.145 \\
\hline
\end{tabular}

Método de extracción: Análisis de componentes principales.

Método de rotación: Normalización Varimax con Kaiser.

La rotación ha convergido en 6 iteraciones.

Fuente: Elaboración propia con base en los resultados obtenidos del AFC.

En la Matriz de Componentes Rotados se toman los datos de la Matriz de Componentes y se aplica un proceso de normalización por el método de 
Varimax, lo que permite una mejor representación de las variables. El efecto de rotación es redistribuir la varianza para obtener un patrón de factores o componentes con mayor significado (Kendall y Buckland, 1990). El criterio de rotación de Varimax se centra en simplificar al máximo los vectores de las columnas de factores (componentes), la simplificación máxima se alcanza al llegar a valores como +1 o al -1 y otras cargas cercanas al 0 , lo que coadyuva a una mejor interpretación de las variables. Los valores van a mostrar una asociación positiva o negativa entre las variables y el componente o una ausencia de asociación si el número arrojado es cero (Santos et al., 2003).

En la Matriz de Componentes Rotados todas las variables quedan claramente definidas en un espacio dimensional. En el primer componente las variables que mejor se explican son el IDH, el I-INGRESO, el PIB PER CÁPITA, el ISAlUd, el I-EDUCACIÓn, la POSICIÓn Del Pib, T-DESEMPleo y el Pib. Para el segundo componente se tiene a MIGRACIÓN, REMESAS Y POBLACIÓN. En el tercer componente se encuentran $3 \times 1$ Migración, $3 \times 1$ mUnicipios y $3 \times 1$ estado. Para el cuarto, quinto, sexto, séptimo y octavo componentes se ubican $3 \times 1$ CTOTAL, INFLACIÓN, PEA, $3 \times 1$ FEDERAL Y TASA DE ANALFABETISMO en ese orden (véase cuadro 3). Las variables mejor representadas en cada una de las dimensiones de la Matriz de Componentes Rotados, son las que se localizan en términos gráficos en sus respectivas dimensiones.

Gráfico de componentes en espacios rotados en tres dimensiones (3D)

El gráfico de componentes en espacios rotados muestra la misma información que la Matriz de Componentes Rotados, exponiendo únicamente las tres dimensiones más representativas, aunque tomando en consideración los efectos de todas las dimensiones estudiadas que en este caso son ocho. Este gráfico refleja la ubicación de las variables en el espacio definido por los componentes, mostrándolas en un espacio ortogonal, pero no como coordenadas sino como vectores en el espacio. Cabe mencionar que la posición que ocupe el vector de cada una de las variables va a posicionar a cada uno de los casos, que para la presente investigación son los treintaiún estados y el Dis- 
trito Federal, con coordenadas únicas de acuerdo a su posición respecto de la variable (ver gráfica 2 ).

\section{GRÁFICA 2}

Gráfico de componentes en espacio rotado

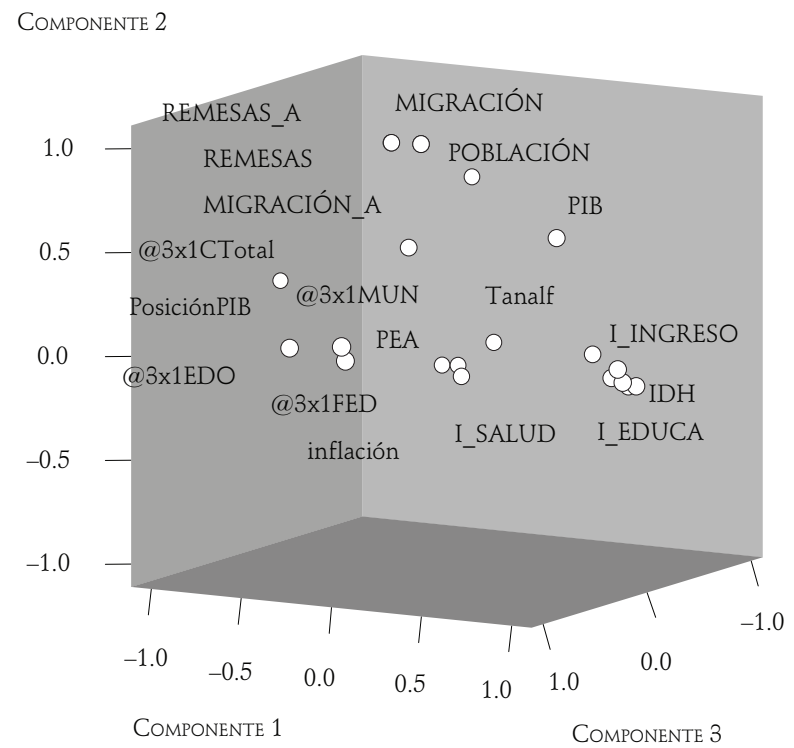

Fuente: Elaboración propia con base en los resultados obtenidos del AFC.

Gráfica de Puntuaciones por estados y variables

La técnica de AFC es interdependiente, es decir, todas las variables se consideran simultáneamente, se relacionan entre sí y forman factores que maximizan la explicación del conjunto de variables, identificando la estructura que existe entre ellas (Santesmases, 1998). El Gráfico de Puntuaciones muestra (en un plano con dos ejes) precisamente ese espacio donde cada caso se encuentra en un punto dado por las coordenadas $(X, Y)$ de las dos dimensiones representadas. Estas coordenadas están influenciadas por la cercanía o leja- 
nía con las variables estudiadas, por lo que este gráfico expresa la correlación de los casos (los estados) con las variables. Es decir, se observa aquí si los casos están afectados, positiva o negativamente, por las variables señaladas. Se examina así, si las variables son contrapuestas, yuxtapuestas, etc.

\section{La migración en México: un anÁlisis bidimensional}

En esta sección se realiza el análisis multidimensional y multivariado a partir de los resultados arrojados por la Matriz de Componentes Rotados, es decir, considerando los factores o componentes donde se encuentran definidas las variables. De manera particular, se pone atención en aquellos factores que por el agrupamiento de variables permiten configurar determinados ejes. Dentro de estos ejes destacan migración, desarrollo y programas $3 \times 1$, los cuales se revisan comparativamente mediante un análisis bidimensional, esto es, referenciando dos factores.

\section{Migración y desarrollo}

En este apartado se analizan los ejes de migración y desarrollo, dimensiones 2 y 1 , ubicándolos en el plano cartesiano con las variables y los estados. La revisión se realiza entonces, a partir de los cuatro cuadrantes, considerando la migración y el desarrollo en México.

\section{Cuadrante I}

Se ubican en este cuadrante los estados que tienen un mayor desarrollo y una alta influencia del fenómeno migratorio. Las variables ubicadas en el eje $X$ que representan la dimensión del desarrollo son el PIB, el Índice de Desarrollo Humano (IDH) y sus componentes el Índice de Salud (I-SALUD), el Índice de Educación (I-EDUCA) y el Índice de Ingreso (I-INGRESO), la tasa de desempleo (T-DeSEMPLEO) y el PIB per cápita. Dentro de las variables situadas en el eje Y 
expresando la dimensión de la migración se tiene a: la POBLACIÓN, la MIGRACIÓN Y las REMESAS.

Los estados que se ubican en este cuadrante son los siguientes:

a) Distrito Federal. Es el que tiene el desarrollo más importante del país. Si bien se encuentra ampliamente influenciado por el fenómeno migratorio, es el estado de Jalisco el que tiene la mayor presencia en esta dirección dentro del cuadrante (véase gráfica 3).

b) Nuevo León. Es después del Distrito Federal la entidad con el mayor desarrollo en México. Es además, el estado en este cuadrante menos relacionado con el primer eje (migración).

c) Jalisco. Dentro del cuadrante es la entidad más vinculada con el fenómeno migratorio, pero es también la que tiene menor nivel de desarrollo.

\section{GRÁFICA 3}

Gráfico de Puntuaciones: Migración y Desarrollo

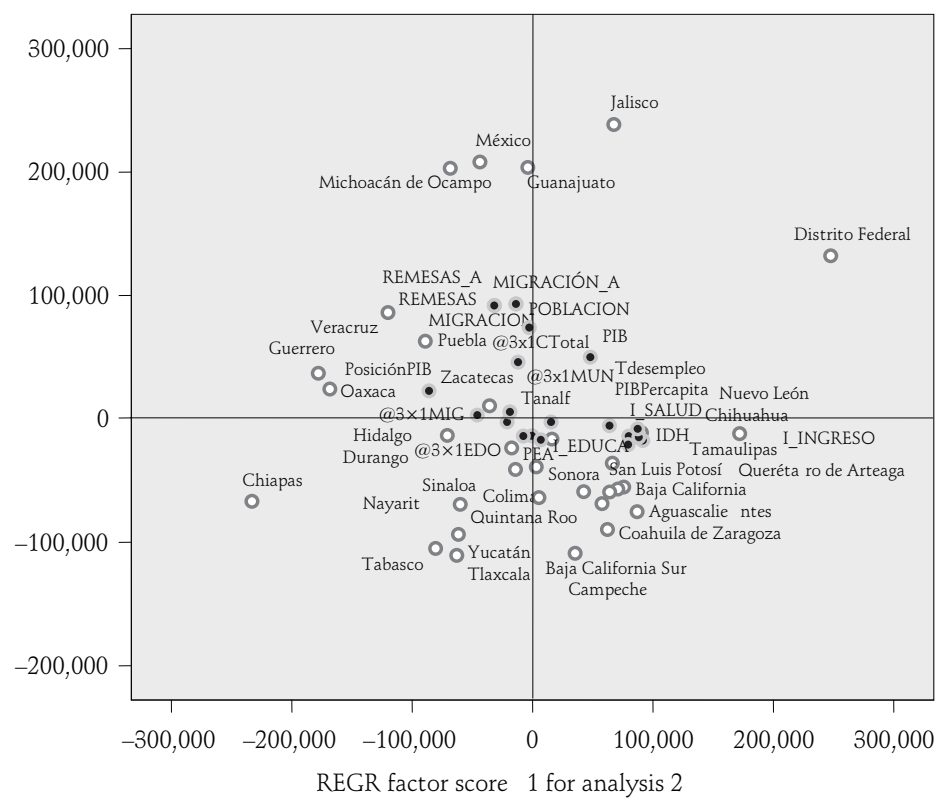

Fuente: Elaboración propia con base en los resultados obtenidos del AFC.

$136 \frac{\text { PRIMER SEMESTRE } 2014}{\text { MIGRACIÓN Y DESARROLLO NÚM. } 22}$ 


\section{Cuadrante II}

Se encuentran en este cuadrante los estados que tienen menor desarrollo, el eje $X$, y una alta influencia del fenómeno migratorio, el eje $Y$.

a) Los estados que muestran una elevada presencia del fenómeno migratorio en este caso son el Estado de México y Michoacán en ese orden. En una posición intermedia se sitúan Veracruz y Puebla. Mientras que Zacatecas, Oaxaca y Guerrero son los que menor influencia tienen de esta dimensión (migración) en el cuadrante.

b) En materia de desarrollo los menos posicionados son Guerrero y Oaxaca, respectivamente. En una posición intermedia están Michoacán, Puebla y Veracruz. En tanto que, dentro de los mejor ubicados en el cuadrante son el Estado de México y Zacatecas.

Cuadrante III

El cuadrante incluye a los estados que tienen menor desarrollo, el eje $X$, y una baja influencia del fenómeno migratorio, el eje $Y$.

a) Con la más baja influencia migratoria se consideran los estados de Yucatán, Tabasco y Quintana Roo. En la posición intermedia están Nayarit, Chiapas y Sinaloa. Con una mayor influencia del fenómeno migratorio en el cuadrante se encuentran Hidalgo y Durango.

b) Es Chiapas el que tiene no sólo el menor desarrollo del cuadrante, sino de todos los estados en general. Existe un grupo de estados que se sitúan en la parte intermedia donde se encuentran Tabasco, Yucatán, Quintana Roo, Hidalgo y Nayarit. Con el mayor desarrollo se ubican en el cuadrante Durango y Sinaloa.

El cuadrante III se distingue porque es aquí donde están las entidades con una mayor problemática en materia de desarrollo en el país. Pero si bien el tener una fuerte presencia del fenómeno migratorio no constituye precisamente una cualidad, se encuentran en este cuadrante, también aquellos estados que no cuentan con una elevada presencia de divisas a través de esta vía para amortiguar los problemas de pobreza. 


\section{Cuadrante IV}

Se localizan aquí los estados con el mayor desarrollo, el eje $X$, y una baja influencia del fenómeno migratorio, el eje $Y$.

a) Con la más baja influencia del fenómeno migratorio se ubican Campeche y Baja California Sur. En la parte media destacan Coahuila, Aguascalientes y Colima. Mientras que, con una mayor influencia migratoria en el cuadrante se encuentran Querétaro, Tamaulipas, Chihuahua, San Luis, Sonora y Baja California.

b) Son los estados de Querétaro, Tamaulipas y Chihuahua los que se sitúan con el mayor nivel de desarrollo en el cuadrante. Existe un amplio grupo ubicados en la parte intermedia donde sobresalen Aguascalientes, Baja California, San Luis Potosí y Coahuila. Con un menor nivel de desarrollo están Sonora, Colima, Baja California Sur y Campeche.

Al revisar la ubicación de los estados en los cuatro cuadrantes, particular atención merecen Guanajuato y Tlaxcala. El primero situado en el eje de la X entre el cuadrante de mayor desarrollo y alta influencia migratoria y en el de menor desarrollo y alta influencia migratoria. Es decir, en materia de desarrollo se sitúa en la parte intermedia y se localiza dentro de los estados con mayor presencia del fenómeno migratorio. Tlaxcala si bien está también en el eje $X$, tiene una posición opuesta a Guanajuato; esto es, está en la parte intermedia en términos de desarrollo pero con una baja influencia de la migración, incluso en general es de las entidades con menor presencia de este fenómeno.

\section{Migración y programas $3 \times 1$}

Un análisis similar al de migración y desarrollo puede efectuarse para los factores o dimensiones migración y programas $3 \times 1$. Estos dos ejes en el plano cartesiano de manera general presentan las siguientes características:

$138 \frac{\text { PRIMER SEMESTRE } 2014}{\text { MIGRACIÓN Y DESARROLLO NÚM. } 22}$ 
a) Eje $X$. Este factor o dimensión da cuenta en este caso de la influencia de la migración por entidad federativa, situándose aquí, aquellos estados con más alta y baja influencia de este fenómeno.

Al igual que en el análisis de migración y desarrollo destacan aquí Michoacán, Jalisco, Guanajuato, el Estado de México, Guerrero, Distrito Federal, Puebla y Zacatecas como las entidades con más alta influencia del fenómeno migratorio. Mientras que sobresalen Tabasco, Baja California Sur, Tlaxcala, Querétaro, Yucatán y Campeche como los estados con la más baja influencia de este fenómeno (véase gráfica 4).

\section{GRÁFICA 4}

Gráfico de Puntuaciones: Migración y Programas $3 \times 1$

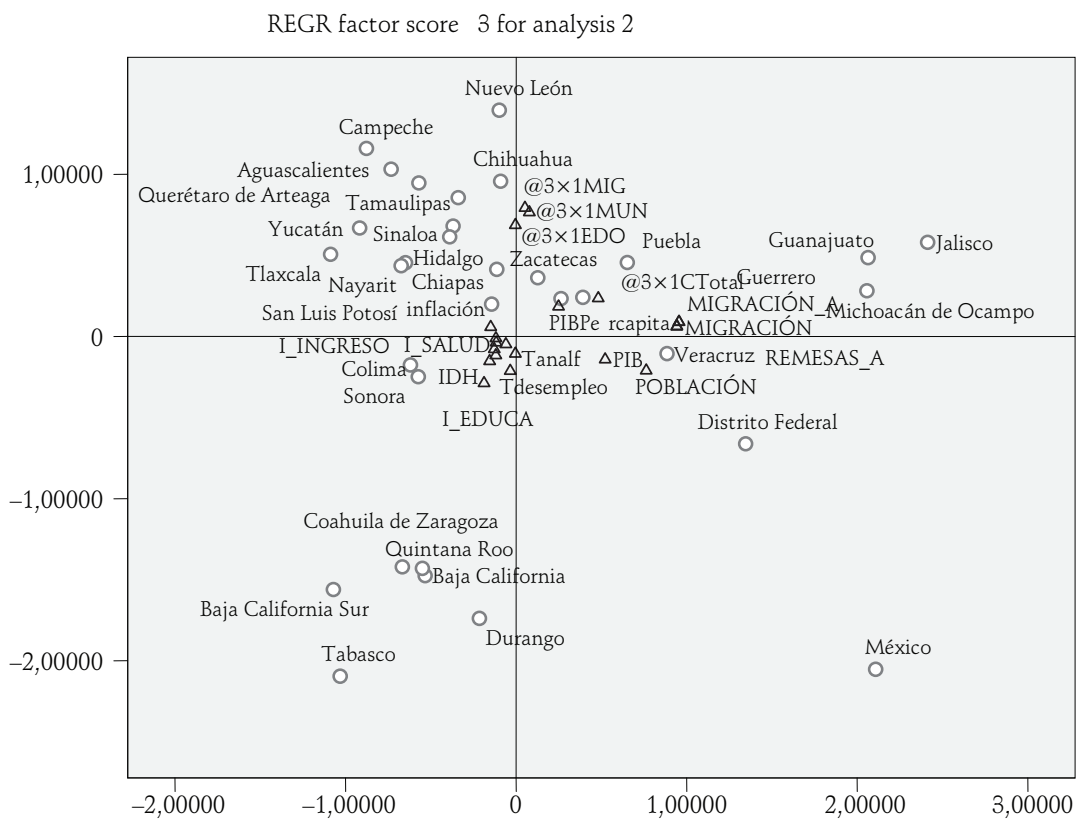

REGR factor score 2 for analysis 2

Fuente: Elaboración propia con base en los resultados obtenidos del AFC. 
b) Eje $Y$. Este factor aglutina a las variables revisadas en este estudio vinculadas con los programas $3 \times 1$. Se localizan aquí entonces, los estados que presentan una mayor $y$ menor incidencia de los programas $3 \times 1$.

Cabría esperar que los estados con una más alta influencia del fenómeno migratorio fueran aquellos en los que se presentara una mayor incidencia de los programas $3 \times 1$. Sin embargo, esto no ocurre en la práctica ya que destacan Nuevo León, Campeche, Aguascalientes, Chihuahua, Querétaro, Tamaulipas, Yucatán y Sinaloa como las entidades donde se tiene una mayor presencia de los programas $3 \times 1$. Mientras que son Tabasco, el Estado de México, Durango, Baja California Sur, Baja California, Quintana Roo, Coahuila y el Distrito Federal, los que tienen una menor incidencia de los programas $3 \times 1$.

Desarrollo y Programas $3 \times 1$

Se analizan aquí los factores o dimensiones desarrollo y programas $3 \times 1$, los que en el plano cartesiano se representan mediante el eje $X$ (desarrollo) y el eje $Y$ (programas $3 \times 1$ ). En términos generales observamos las siguientes características:

a) Eje $X$. En este factor o dimensión se agrupan las variables vinculadas con los niveles de desarrollo. Se encuentran entonces en este eje, los estados con mayor y menor desarrollo.

Sobresalen con un mayor desarrollo el Distrito Federal, Nuevo León, Jalisco y San Luis Potosí. En tanto que, con el menor desarrollo tenemos a Chiapas, Michoacán, Oaxaca, Guerrero, Veracruz y Puebla (ver gráfica 5).

Eje $Y$. Este factor o dimensión considera las variables vinculadas con los programas $3 \times 1$. Están en consecuencia aquí las entidades donde los programas $3 \times 1$ tienen una mayor o menor presencia. Los estados que destacan por tener una mayor presencia de los programas $3 \times 1$ son Nuevo León, Que-

$140 \frac{\text { PRIMER SEMESTRE } 2014}{\text { MIGRACIÓN Y DESARROLLO NÚM. } 22}$ 


\section{GRÁFICA 5}

Gráfico de Puntuaciones: Desarrollo y Programas $3 \times 1$

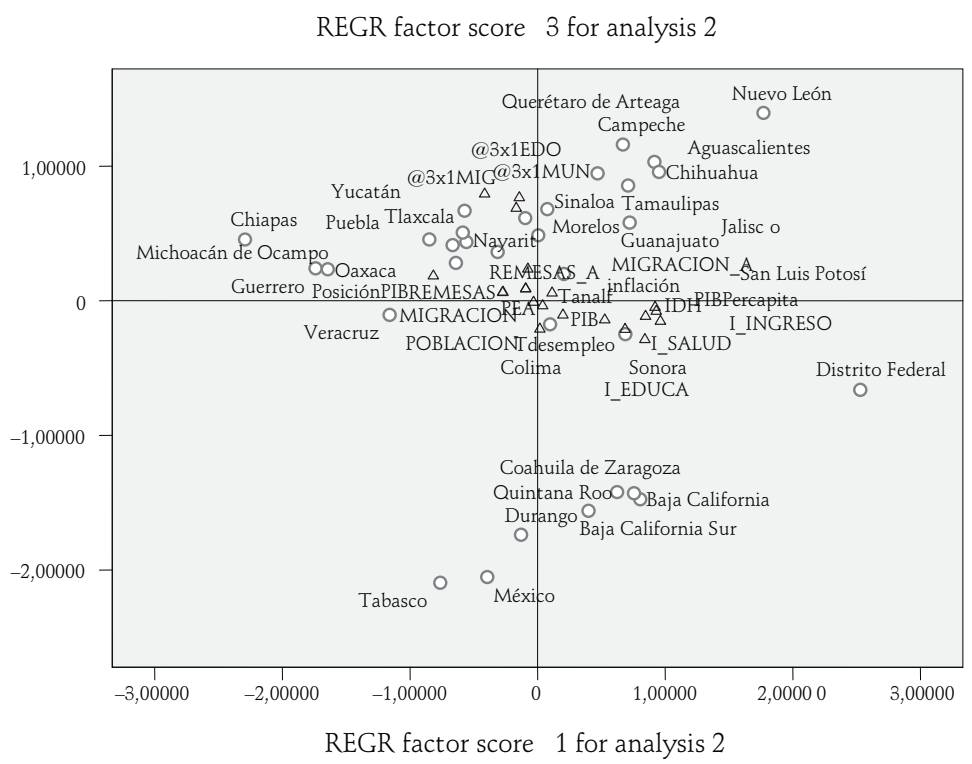

Fuente: Elaboración propia con base en los resultados obtenidos del AFC.

rétaro, Campeche, Aguascalientes, Chihuahua, Tamaulipas y Sinaloa. Mientras que con una menor presencia de estos programas tenemos a Tabasco, el Estado de México, Durango, Baja California Sur, Baja California, Quintana Roo, Coahuila y el Distrito federal.

\section{Conclusiones}

La metodología del AFC permite estudiar la dependencia entre variables, definiendo para esto un número de dimensiones comunes, denominadas componentes o factores. Esta técnica, además, permite realizar estudios de proximidad entre las modalidades de una misma variable, evaluando su homogeneidad y sustituibilidad. El AFC mediante el tratamiento multidimensional y multivariable, establece coordenadas influenciadas por la cerca- 
nía o lejanía de las variables en estudio. Se expresa así, la correlación de los casos (estados) con las variables.

Los resultados del AFC se encuentran en la Matriz de Componentes y en la Matriz de Componentes Rotados, las cuales indican la dirección en el espacio donde se sitúan las variables, así como sus niveles de correlación. Si bien la Matriz de Componentes en un primer momento permite identificar las variables mejor explicadas, presenta algunos problemas al definir las variables, los que se resuelven en un segundo momento al trabajar con la Matriz de Componentes Rotados. Esto es, en la Matriz de Componentes Rotados todas las variables quedan claramente definidas en un espacio dimensional. El Gráfico de Puntuaciones muestra en un plano con dos ejes precisamente ese espacio donde cada caso se encuentra en un punto dado por las coordenadas $(X, Y)$ de las dos dimensiones representadas.

El análisis multivariante y multidimensional se realiza a partir de los resultados obtenidos de la Matriz de Componentes Rotados. Esto es, considerando los factores o componentes donde se encuentran definidas las variables. Se destacan aquellos factores que por el agrupamiento de variables permiten configurar determinados ejes. Dentro de estos ejes destacan el de migración, el de desarrollo y el de programas $3 \times 1$, los cuales se revisan comparativamente mediante un análisis bidimensional; es decir, referenciando dos factores.

El primer análisis bidimensional se realiza para los ejes migración y desarrollo, revisándose la posición de las entidades federativas en los cuatro cuadrantes, así como la mayor o menor influencia de las variables que definen estos ejes:

1) En el primer cuadrante se encuentran los estados que presentan un mayor desarrollo y una alta influencia del fenómeno migratorio. Sobresale el Distrito Federal con el desarrollo más importante del país seguido de Nuevo León. Mientras que Jalisco es el que tiene la más alta influencia del fenómeno migratorio a la par que es la entidad con el menor desarrollo del cuadrante.

2) En el segundo cuadrante se ubican los estados con menor desarrollo y una alta influencia de la migración. Destacan Guerrero y Oaxaca como los menos

$142 \frac{\text { PRIMER SEMESTRE } 2014}{\text { MIGRACIÓN Y DESARROLLO NÚM. } 22}$ 
desarrollados y el Estado de México y Michoacán con la más elevada presencia del fenómeno migratorio.

3) En el tercer cuadrante se incluyen los estados con el menor desarrollo y una baja influencia del fenómeno migratorio. Chiapas es el que tiene no sólo el menor desarrollo del cuadrante, sino de todos los estados en general. Sobresalen asimismo, Yucatán, Tabasco y Quintana Roo con la más baja influencia migratoria.

4) En el cuarto cuadrante se localizan los estados de mayor desarrollo y una baja influencia migratoria. Son de esta manera, Querétaro, Tamaulipas y Chihuahua los que se sitúan con el mayor nivel de desarrollo. Mientras que, Campeche y Baja California Sur se distinguen por su menor presencia de la migración.

El segundo análisis bidimensional se efectúa para los ejes migración y programas $3 \times 1$. En materia de migración destacan Michoacán, Jalisco, Guanajuato, el Estado de México, Guerrero, Distrito Federal, Puebla y Zacatecas por su alta influencia del fenómeno migratorio. Son a la vez, Tabasco, Baja California Sur, Tlaxcala, Querétaro, Yucatán y Campeche los estados con la más baja influencia de este fenómeno.

Si bien los estados con una más alta influencia del fenómeno migratorio podrían marcar la pauta en relación a los programas $3 \times 1$, en la práctica esto no ocurre, ya que son Nuevo León, Campeche, Aguascalientes, Chihuahua, Querétaro, Tamaulipas, Yucatán y Sinaloa las entidades donde se tiene una mayor presencia de estos programas. En tanto que, Tabasco, el Estado de México, Durango, Baja California Sur, Baja California, Quintana Roo, Coahuila y el Distrito Federal, son los que tienen una menor incidencia de los programas $3 \times 1$.

En el tercer análisis bidimensional que considera los ejes desarrollo y programas $3 \times 1$, confirma los resultados obtenidos del primero, migración y desarrollo, y del segundo, migración y $3 \times 1$, análisis bidimensional. Esto es, las entidades conservan sus posiciones en el ámbito nacional en los factores de desarrollo y programas $3 \times 1$. 
Las asimetrías que las entidades federativas muestran en materia de desarrollo, bienestar social y migración, identificadas en este estudio metodológicamente a través del AFC, conllevan a la necesidad inmediata de avanzar en torno a políticas públicas más efectivas para enfrentar los retos del desarrollo nacional.

\section{Bibliografía}

Adams, R. H. (2005), «Remittances, Household Expenditure and Investment in Guatemala», World Bank Policy Research, Working Paper No. 3532.

(1998), «Remittances, Investment and Rural Asset Accumulation in Pakistan», Economic Development and Cultural Change, vol. 47.

(1991), "The Economic uses and Impact of International Remittances in Rural Egypt», Economic Development and Cultural Change, vol. 39.

BANXICO (2010), «Índice Nacional de precios al Consumidor por área metropolitana y principales ciudades».

Batista, J. y S. Joan (1997), Análisis de correspondencias y técnicas de clasificación: Su interés para la investigación en las ciencias sociales y del comportamiento, Barcelona, Universidad de Barcelona.

Callealta, F. J. (2005), A New Measure of Dissimilarity Between Distributions: Aplication to the Analysys of Income Distributions Convergence in the European Union, Madrid, Departamento de Estadística, Estructura Económica y oEI.

Cattell, R. B. (1966), "The Scree Test for the Number of Factors», Multivariate Behavioural Reseach, vol. 1, núm. 2.

Cox, T. F. and M. A. Cox (1994), Multidimensional Scaling, London, Chapman \& Hall. ICTEM (2010), Índice de Competitividad Turísitica de los Estados Mexicanos. Tecnológico de Monterrey.

INEGI (2010), Encuesta Nacional de Ocupación y Empleo; Indicadores Estratégicos de Ocupación y Empleo, INEGI.

(2010), Sistema de Cuentas Nacionales de México, INEGI.

Kendall, M. and W. R. Buckland (1990), Dictionary of Statistical Term, Edinburgh, Oliver and Boyd.

Kennedy, P. (1985), A Guide to Econometrics, Cambridge, The mit Press.

$\mathbf{1 4 4} \frac{\text { PRIMER SEMESTRE } 2014}{\text { MIGRACIÓN Y DESARROLLO NÚM. } 22}$ 
KIM, J., and C. Mueller (1978), An introduction to factor analysis: What it is and how to do it, Beverly Hills, Sage.

Kruskal, J. and M. Wish, M. (1981), Multidimensional Scaling, Beverly Hills Sage Publications.

LucAs, R. E. B. (1987), «Emigration to South Africa's mines», American Economic Review, vol. 77, núm. 3.

McKenzie, D. and H. Rapoport (2005), "Network Effects and the Dynamics of Migration and Inequality: Theory and Evidence from Mexico», Bureau for Research in Economic Analysis of Development (BREAD), Working Paper No. 063, April.

Mrouel, S., E. Bigné, J. P. Lévy, A. Cuenca, y M. Miquel (1997), Investigación de mercados, Madrid, McGraw Hill.

MorA, J. and J. E. Taylor (2005), «Determinants of Migration, Destination and Sector Choice: Disentangling Individual, Household and Community Effects», in Ça-glar Özden and Maurice Schiff (eds.), International Migration, Remittances, and the Brain Drain, New York, Palgrave Macmillan.

Munshi, K. (2003), "Networks in the Modern Economy: Mexican Migrants in the U.S. Labor Market», Quarterly Journal of Economics, vol. 18, núm. 2.

Pérez, L. C. (2006), Técnicas de Análisis Multivariante de Datos, Barcelona, Pearson/ Prentice Hall.

PNUD (2010), Índice de Desarrollo Humano IDH, PNUD.

Rodríguez, O., W. Castillo, E. Diday y J. González (2003), Correspondence Factorial Análisis for Symbolic Multi-Valued Variables, Universidad de Costa Rica.

Rozelle, S., J. E. Taylor and A. de Brauw (1999), "Migration, Remittances, and Agricultural Productivity in China», American Economic Review, vol. 89, núm. 2.

SAdoulet, E., A. de Janvry and B. Davis (2001), "Cash Transfer Programs with Income Multipliers: Procampo in Mexico", World Development, vol. 29, no 6.

Santesmases, M. (1998), Marketing. Conceptos y Estrategias, Madrid, Ediciones Pirámide. Santos, J. A., A. Muñoz, P. Juez, y P. Cortiñas (2003), Diseño de encuestas para estudios de mercado, Madrid, Centro de Estudios Ramón Areces. 
